7th International Workshop on Astronomy and

Relativistic Astrophysics (IWARA 2016)

International Journal of Modern Physics: Conference Series

Vol. 45 (2017) 1760011 (5 pages)

(C) The Author(s)

DOI: $10.1142 / S 2010194517600114$

\title{
Dark Matter in a Bimetric Portal
}

\author{
Dimiter Hadjimichef \\ Instituto de Física \\ Universidade Federal do Rio Grande do Sul (UFRGS) \\ Av. Bento Gonçalves, 9500, 91501-970 Porto Alegre, RS, Brazil \\ dimiter.hadjimichef@ufrgs.br
}

Published 15 August 2017

\begin{abstract}
One of the great mysteries in current physics is the nature of dark matter. Arguments are brought forward to consider a form dark matter, manifested as a broken local dual symmetry of a bimetric spin-2 theory in the Fierz representation.
\end{abstract}

Keywords: Dark Matter, Modified Theories of Gravity, Dual Symmetry.

PACS numbers: 95.35.+d, 04.50.Kd

\section{Introduction}

The enigma of dark matter remains unsolved. Over the years, dark matter studies have split into two major branches: one as an astrophysical phenomena and another as particle physics problem. In particle physics, the dark matter appears as an extension of the Standard Model of particles. Its search has introduced many candidates that converge to a variety of interesting and plausible candidates namely the weakly-interacting massive particles (WIMPs). ${ }^{1}$ In general they are present in theories of weak-scale physics beyond the Standard Model and give rise to appropriate relic abundance. Calculations have shown that stable WIMPs can remain from the earliest moments of the Universe in sufficient number to account for a significant fraction of relic dark matter density. This raises the hope of detecting relic WIMPs directly by observing their elastic scattering on targets. In this dark matter zoo many different types of particles have been proposed and their properties theoretically studied, but experimentally there has been no conclusive evidence of particle dark matter to the present day.

If particle searches fail to reveal new physics, then could dark matter be some form of exotic matter only coupled to gravity? In the present experimental status,

This is an Open Access article published by World Scientific Publishing Company. It is distributed under the terms of the Creative Commons Attribution 4.0 (CC-BY) License. Further distribution of this work is permitted, provided the original work is properly cited. 


\section{Hadjimichef}

dark matter is only observed in the large scale structure, i.e. where there is an abundant amount of ordinary matter, in contrast to particle searches where the mass density is low and the scale is small. This approach has been tried in some cases such as disformal relations appearing in cosmological models of alternative dark matter, in particular TeVeS (see Ref. ${ }^{2}$ ), or in mimetic dark matter where the physical metric $g_{\mu \nu}^{\text {phys }}$ is considered to be a function of a scalar field $\phi$ and a fundamental metric $g_{\mu \nu}$ (see Ref. ${ }^{3}$ ). The physical metric is invariant with respect to a Weyl transformation, resulting in a model which is a conformal extension of General Relativity. In this sense, could there exist other symmetries that, to be manifest, would require extra fields generally interpreted as dark matter?

\section{Spin-2 Field Description in Minkowski Background}

As well known that in the description of a spin-2 tensor field one can use either a second-order representation that deals with a symmetric second-order tensor $h_{\mu \nu}$ or a first-order one, in terms of a third-order tensor $\Phi_{\mu \nu \alpha}$ (the Fierz representation), which we shall consider the case of spin-2 in the Minkowski background. We shall follow closely the formalism developed in Ref. ${ }^{4}$ First define a three-index tensor $\Phi_{\alpha \beta \mu}$ which is anti-symmetric in the first pair of indices and obeys the cyclic identity, that is

$$
\Phi_{\alpha \mu \nu}+\Phi_{\mu \alpha \nu}=0 \quad ; \quad \Phi_{\alpha \mu \nu}+\Phi_{\mu \nu \alpha}+\Phi_{\nu \alpha \mu}=0 .
$$

This last expression means that the dual of $\Phi_{\alpha \mu \nu}$ is trace-free and one can define the dual tensor ${ }^{*} \Phi$ is defined as

$$
{ }^{*} \Phi^{\mu \nu \alpha}=\frac{1}{2} \varepsilon^{\mu \nu \rho \sigma} \eta^{\alpha \beta} \Phi_{\rho \sigma \beta} .
$$

written in terms of the completely anti-symmetric object $\varepsilon_{\alpha \beta \mu \nu}$. The necessary and sufficient condition that $\Phi_{\alpha \mu \nu}$ represents an unique spin-2 field is

$$
\partial_{\alpha}^{*} \Phi^{\alpha(\mu \nu)}=0
$$

where $A_{(\mu \nu)} \equiv A_{\mu \nu}+A_{\nu \mu}$. A tensor that satisfies conditions (1) and (3) is called a Fierz tensor. Condition (3) implies that there exists a symmetric second order tensor $h_{\mu \nu}$ such that we can write

$$
2 \Phi_{\alpha \mu \nu}=\partial_{\mu} h_{\nu \alpha}-\partial_{\alpha} h_{\nu \mu}+\eta_{\mu \nu}\left(\partial_{\alpha} h-\partial_{\lambda} h_{\alpha}{ }^{\lambda}\right)-\eta_{\alpha \nu}\left(\partial_{\mu} h-\partial_{\lambda} h_{\mu}{ }^{\lambda}\right) .
$$

with $h=h^{\mu}{ }_{\mu}$. Now, from (4) one can show that

$$
\partial_{\alpha} \Phi_{(\mu \nu)}^{\alpha}=-G_{\mu \nu}^{L},
$$

where $G^{L}{ }_{\mu \nu}$ is the linearized Einstein operator defined in terms of the symmetric tensor $h_{\mu \nu}$. Now if one defines

$$
A_{\mu \nu}=h_{\mu \nu}-\frac{1}{2} \eta_{\mu \nu} h,
$$

the Fierz tensor in (5) can be written in terms of a new tensor $F_{\alpha \mu \nu}$ defined as

$$
F_{\alpha \mu \nu}=\partial_{\alpha} A_{\mu \nu}-\partial_{\mu} A_{\alpha \nu}-\eta_{\alpha \nu} \partial_{\beta} A_{\mu}^{\beta}+\eta_{\mu \nu} \partial_{\beta} A_{\alpha}^{\beta},
$$


with $\Phi_{\alpha(\mu \nu)}=-F_{\alpha \mu \nu}$, Eq. (5) reduces to

$$
\partial_{\alpha} F_{\mu \nu}^{\alpha}=G_{\mu \nu}^{L} .
$$

A gauge fixing condition $\partial_{\beta} A^{\beta}{ }_{\mu}=0$ can be applied and (6) reduces to

$$
F_{\alpha \mu \nu}=\partial_{\alpha} A_{\mu \nu}-\partial_{\mu} A_{\alpha \nu}
$$

The linear equation of gravity in (7), in the vacuum, is invariant under the following dual transformation

$$
F^{\mu \nu \alpha} \rightarrow \cos \theta F^{\mu \nu \alpha}+\sin \theta^{*} F^{\mu \nu \alpha} ;{ }^{*} F^{\mu \nu \alpha} \rightarrow-\sin \theta F^{\mu \nu \alpha}+\cos \theta^{*} F^{\mu \nu \alpha}
$$

where $\theta$ is the dual angle.

\section{Dual Symmetry and Dark Matter}

A bimetric gravity refers to a class of modified mathematical theories of gravity in which two metric tensors are used instead of one. If the second metric tensor is related to a second Fierz tensor $C^{\mu \nu \alpha}$, such that

$$
C^{\mu \nu \alpha}=\partial^{\mu} C^{\nu \alpha}-\partial^{\nu} C^{\mu \alpha}
$$

independent of $F^{\mu \nu \alpha}$, an interesting alternative arises if subject to transform by (8):

$$
F^{\mu \nu \alpha} \rightarrow \cos \theta F^{\mu \nu \alpha}+\sin \theta C^{\mu \nu \alpha} ; C^{\mu \nu \alpha} \rightarrow-\sin \theta F^{\mu \nu \alpha}+\cos \theta C^{\mu \nu \alpha},
$$

implementing an extended version of dual symmetry. Transformations (10) reduce to (8) when the dual constraint

$$
C^{\mu \nu \alpha}={ }^{*} F^{\mu \nu \alpha},
$$

is used. The dual-symmetric formalism acquires a particularly abbreviated form if we introduce the complex potential $G^{\mu \nu}$ defined by

$$
G^{\mu \nu}=A^{\mu \nu}+i C^{\mu \nu}
$$

where the corresponding field tensor $G^{\mu \nu \alpha}$ is written as

$$
G^{\mu \nu \alpha}=\partial^{\mu} G^{\nu \alpha}-\partial^{\nu} G^{\mu \alpha}=F^{\mu \nu \alpha}+i C^{\mu \nu \alpha} .
$$

The two vacuum equations originated from the linear gravity equations are now combined into one complex equation

$$
\partial_{\mu} G^{\mu \nu \alpha}=0 .
$$

Extended dual symmetry transformations (10) are implemented in a very compact form as phase transformations, global $U(1)$ symmetry, of these fields:

$$
G^{\mu \nu} \rightarrow e^{-i \theta} G^{\mu \nu}
$$




\section{Hadjimichef}

which implies that the field tensor transforms as

$$
G^{\mu \nu \alpha} \rightarrow e^{-i \theta} G^{\mu \nu \alpha},
$$

and maintains (11) invariant.

How can a form of dark matter emerge from dual symmetry of linear gravity? As presented until now, dual symmetry appears as a global symmetry of the vacuum equations of linear gravity, defined by a single constant parameter, the dual angle $\theta$. If this parameter is no longer a constant, but a local space-time field $\theta(x)$, DuSy is promoted to a local symmetry. This implies in substituting $\theta \rightarrow q \theta(x)$ in (12), obtaining

$$
G^{\mu \nu} \rightarrow e^{-i q \theta(x)} G^{\mu \nu}
$$

where $q$ is a new charge in the problem. Under the transformation (13) the field tensor $G^{\mu \nu \alpha}$ transforms as

$$
G^{\mu \nu \alpha} \rightarrow e^{-i q \theta(x)}\left(G^{\mu \nu \alpha}-i q \Theta^{\mu \nu \alpha}\right),
$$

where

$$
\Theta^{\mu \nu \alpha}=\left(\partial^{\mu} \theta\right) G^{\nu \alpha}-\left(\partial^{\nu} \theta\right) G^{\mu \alpha} .
$$

To obtain a transformation for the field tensor that is similar to $G^{\mu \nu \alpha} \rightarrow e^{-i \theta} G^{\mu \nu \alpha}$, one can define

$$
K^{\mu \nu \alpha}=q\left(V^{\mu} G^{\nu \alpha}-V^{\nu} G^{\mu \alpha}\right),
$$

where $V^{\mu}$ is an additional complex vector field, with a standard form of gauge transformation $V^{\mu} \rightarrow V^{\mu}-i \partial^{\mu} \theta$. Then under local DuSy (13), $K^{\mu \nu \alpha}$ transforms as

$$
K^{\mu \nu \alpha} \rightarrow e^{-i q \theta(x)}\left(K^{\mu \nu \alpha}-i q \Theta^{\mu \nu \alpha}\right) .
$$

Combining $G^{\mu \nu \alpha}$ and $K^{\mu \nu \alpha}$ into a new tensor field $B^{\mu \nu \alpha}$, given by

$$
B^{\mu \nu \alpha}=G^{\mu \nu \alpha}-K^{\mu \nu \alpha},
$$

it is simple to verify that under the transformation (13),

$$
B^{\mu \nu \alpha} \rightarrow e^{-i q \theta(x)} B^{\mu \nu \alpha} .
$$

Now if the field tensor $B^{\mu \nu \alpha}$ is to be considered as the new gravitational field satisfying an equation that replaces (11), one finds that under the local dual transformation (13):

$$
\partial_{\mu} B^{\mu \nu \alpha} \rightarrow e^{-i q \theta(x)}\left(\partial_{\mu}-i q \partial_{\mu} \theta\right) B^{\mu \nu \alpha} .
$$

A covariant derivative can be defined as $D_{\mu}=\partial_{\mu}+q V_{\mu}^{*}$, such that, a local DuSy invariant equation can finally be obtained

$$
D_{\mu} B^{\mu \nu \alpha}=0 \text {. }
$$




\section{Conclusions}

In the vacuum, equation (20) is the new fundamental field equation which is totally indistinguishable from the original vacuum equation (11). In the presence of matter this symmetry is broken. The vector field $V_{\mu}$ couples only to gravity and, eventually, to the fields of the dark sector. Detection of this type of dark matter is a subtle issue: (i) anomalous cooling of the pulsating white dwarf star; (ii) Compton-like scattering of $V_{\mu}$ on dark $\chi$ fermions, in a process of the type $V+\chi \rightarrow g+\chi$, with a final conversion into detectable gravitational radiation $g$.

\section{Acknowledgments}

This research was supported by Universidade Federal do Rio Grande do Sul (UFRGS).

\section{References}

1. G. Bertone, D. Hooper and J. Silk, Phys. Rep. 405, 279 (2005) [arXiv:hep$\mathrm{ph} / 0404175](2004)$.

2. M. Zumalacárregui, T. S. Koivisto, D. F. Mota and P. Ruiz-Lapuente, JCAP 038, 1005 (2010).

3. A. H. Chamseddine and V. Mukhanov, JHEP 011, 135 (2013).

4. M. Novello and R. P. Neves, Class. Quant. Grav. 19, 5335 (2002). 